\title{
Estresse Ocupacional em Profissionais de Saúde: Um Estudo com Enfermeiros Portugueses ${ }^{1}$
}

\author{
A. Rui Gomes ${ }^{2}$ \\ José Fernando Cruz \\ Susana Cabanelas \\ Universidade do Minho
}

\begin{abstract}
RESUMO - Este trabalho analisa o estresse ocupacional em 286 enfermeiros de hospitais e centros de saúde portugueses. Avaliaram-se as fontes de estresse, o burnout, os problemas de saúde física, a satisfação e a realização profissional. Os resultados apontaram 30\% de enfermeiros com experiências significativas de estresse e 15\% com problemas de exaustão emocional. As análises de regressão múltipla apontaram maior capacidade preditiva das dimensões de estresse na exaustão emocional, na saúde física, na satisfação e na realização profissional. As análises comparativas evidenciaram maiores problemas de estresse e reações mais negativas ao trabalho nas mulheres, nos enfermeiros mais novos e com menor experiência, nos trabalhadores com contratos a prazo, nos profissionais que realizam trabalho por turnos e nos que trabalham mais horas.
\end{abstract}

Palavras-chave: enfermagem; estresse ocupacional; burnout; saúde; satisfação.

\section{Occupational Stress in Health Professionals: A Study with Portuguese Nurses}

\begin{abstract}
This work analyses occupational stress in 286 nurses from hospitals and health care centres in Portugal. The following dimensions were evaluated: stress, burnout, physical health problems, satisfaction and professional fulfilment. Results revealed significant stress experiences in $30 \%$ of the professionals, and emotional exhaustion problems in $15 \%$. Multiple regression analysis pointed out stress as an important predictor of emotional exhaustion, physical health, satisfaction and professional fulfilment. Comparative analysis suggested more occupational stress and professional negative experiences in the following groups: female nurses, younger and less experienced nurses, those with short-term working contracts, nurses working on a shift-basis system, and nurses working during long hours.
\end{abstract}

Keywords: nursing; occupational stress; burnout; health; satisfaction.

O problema do estresse ocupacional em profissionais da saúde e em particular em enfermeiros é um tema contemporâneo de debate e investigação. Assim, os estudos têm vindo a evidenciar que os enfermeiros representam uma classe profissional particularmente exposta a elevados níveis de pressão e estresse (Bourbonnais, Comeau, Vézina \& Guylaine, 1998; Butterworth, Carson, Jeacock, White \& Clements, 1999; McIntyre, McIntyre \& Silvério, 1999; Murofuse, Abranhes \& Napoleão, 2005; Payne \& Firth-Cozens, 1987). A título ilustrativo, Petterson, Arnetz, Arnetz e Horte (1995) verificaram, em um trabalho levado a cabo com enfermeiros suecos, valores superiores a $80 \%$ de estresse elevado a muito elevado.

Devido a essas indicações, os investigadores têm procurado verificar quais os potenciais efeitos negativos na saúde e bem-estar desses profissionais, descrevendo-se consequências em vários níveis como, por exemplo, menor satisfação

1 Trabalho financiado pelo Centro de Investigação em Psicologia (CIPsi), Universidade do Minho, Portugal. Os autores agradecem as valiosas correções e sugestões fornecidas pelos revisores anônimos designados pelo Conselho Editorial da Revista Psicologia: Teoria e Pesquisa, que ajudaram a melhorar e enriquecer a versão final deste artigo.

2 Endereço para correspondência: Universidade do Minho, Escola de Psicologia. Campus de Gualtar. Braga, Portugal. CEP 4710-057. E-mail: rgomes@iep.uminho.pt.
(Blegen, 1993), mais queixas de natureza física e psicológica (Cooper, Dewe \& O’Driscoll, 2001; Hillhouse \& Adler, 1997; Marshall \& Barnett, 1993) e maior absentismo (Borda \& Norman, 1997; Sauter, Hurrell \& Cooper, 1989).

Para além desse nível individual de análise, o interesse pelo estudo desses profissionais é ainda justificado pela natureza dos serviços que prestam, uma vez que a qualidade e eficácia do seu trabalho pode ter um impacto decisivo na saúde dos pacientes. Na realidade, e contrariamente a muitas outras profissões, a tomada de decisão é particularmente decisiva nessa ocupação laboral, pois a opção por um procedimento de tratamento e/ou ajuda errados pode não só piorar o estado do paciente como, em casos limite, levar à sua morte. Como referem Melo, Gomes e Cruz (1997), uma fonte de tensão especial para os profissionais de saúde consiste na possibilidade do seu próprio estresse poder gerar pressão e mal-estar naquele que é o centro da sua atenção: o doente. Adicionalmente, devem ainda ser referidos os perigos para a própria saúde do profissional, uma vez que algumas das patologias dos doentes são de natureza infecciosa (e.g., sida), colocando desafios constantes e pressão ao exercício dessa profissão.

Tendo por base essas indicações, torna-se difícil explicar qual a razão da área da saúde ser uma das menos estudadas no conjunto dos vários domínios laborais, apesar de envolver 
profissões consideradas de alto risco em termos de estresse (McIntyre, 1994). Por outro lado, e como referem Melo, Gomes e Cruz (1997), embora a área do estresse seja uma das mais produtivas na Psicologia em termos de investigação, ainda faltam estudos dedicados à análise das suas causas, efeitos, prevalência e incidência, sendo tal situação mais evidente em Portugal, onde a investigação é ainda escassa.

Tendo por base essas limitações, propomos neste trabalho contribuir para uma melhoria do conhecimento acerca do estresse ocupacional numa amostra constituída por profissionais de enfermagem portugueses. Em termos de análise, a nossa opção foi no sentido de assumir o estudo do estresse a partir da observação das fontes de pressão associadas ao exercício profissional, procurando-se depois estudar as relações existentes com os níveis de saúde física, de satisfação, de realização profissional e de esgotamento (burnout). Assim sendo, o trabalho levado a cabo pretendeu atingir as seguintes metas:

a) Apresentar metodologias de avaliação do estresse ocupacional, em termos das características psicométricas e de validação para os profissionais da área da saúde;

b) Examinar as fontes de estresse, a prevalência de burnout, os níveis de satisfação e realização profissional e os principais problemas de saúde física dos enfermeiros;

c) Analisar as relações entre estresse, burnout, saúde física, satisfação e realização profissional;

d) Definir as variáveis preditoras da experiência de burnout, saúde física, satisfação e realização profissional; e

e) Observar o impacto de algumas variáveis demográficas e indicadores concretos da situação profissional na experiência de estresse, burnout, saúde física, satisfação e realização profissional manifestada pelos enfermeiros.

\section{Método}

\section{Participantes}

Participaram neste estudo 286 enfermeiros, que trabalhavam em diferentes instituições de saúde (hospitais e centros de saúde) da região norte de Portugal, sendo 224 do sexo feminino $(78,3 \%)$ e 62 do sexo masculino $(21,7 \%)$. As idades variaram entre 22 e 66 anos, com uma média de praticamente 36 anos. A formação dos enfermeiros distribuiu-se pela licenciatura $(92,6 \%)$, seguindo-se o bacharelato $(6 \%)$, o mestrado $(1,1 \%)$ e, por fim, outros graus de formação $(0,3 \%)$. No que se refere ao vínculo profissional, 45 profissionais $(15,7 \%)$ encontravam-se com contrato a prazo (vínculo precário), 149 (52,1\%) faziam parte dos quadros dos respectivos locais de trabalho (vínculo estável) e 92 (32,2\%) não forneceram informação a esse respeito. No que se refere ao horário de trabalho, $157(54,9 \%)$ dos participantes trabalhavam em turnos e $37(12,9 \%)$ seguiam o horário diurno normal. Em termos da área de serviço, foram 15 as especialidades encontradas, sendo de salientar a presença mais significativa na emergência hospitalar $(n=65,22,7 \%)$, clínica geral $(n=44,15,4 \%)$ e cirurgia $(n=42,14,7 \%)$. A média de anos de serviço variou entre 1 e 32 anos $(M=9,7)$ e o número de horas de trabalho por semana apresentou uma grande variabilidade de tempo (30 a 80 horas), com uma média ligeiramente abaixo de 39 horas.

\section{Instrumentos}

Foi administrado a todos os profissionais um conjunto de instrumentos destinados a obter informações acerca das variáveis em análise neste estudo.

Questionário demográfico. Esse instrumento, para além de obter informações acerca do sexo, da idade, do estado civil e do número de filhos, recolheu dados relativos à formação acadêmica, às características e condições gerais de trabalho (e.g., contexto profissional, situação contratual, trabalho por turnos, especialidade de trabalho, experiência profissional e horas de trabalho por semana).

Questionário de Estresse nos Profissionais de Saúde (QSPS). Esse instrumento foi desenvolvido a partir dos trabalhos originais de Cruz e Melo (1996) e Gomes, Melo e Cruz (2000) com psicólogos portugueses, realizando-se a adaptação dos itens de avaliação das fontes de estresse de acordo com os profissionais da área da saúde a que se destina o instrumento (e.g., enfermeiros e médicos). Assim sendo, o questionário compreende duas partes distintas. Na parte inicial, foi proposto aos profissionais a avaliação do nível global de estresse que experimentam na sua profissão, por meio de um único item ( $0=$ Nenhum estresse; $4=$ Elevado estresse). $\mathrm{Na}$ segunda parte, foram avaliadas as potenciais fontes de estresse no exercício profissional (independentemente do contexto, área e/ou domínio de trabalho dos profissionais de saúde). Para tal, foram propostos 70 itens relativos às potenciais fontes de estresse no trabalho, sendo respondidos numa escala tipo Likert de cinco pontos $(0=$ Nenhum estresse; 4=Elevado estresse). Mais à frente, na seção de resultados, são apresentados os resultados da validação do questionário, em termos da estrutura fatorial e respectivas dimensões de avaliação.

Inventário de Burnout de Maslach - Prestadores de Serviços Humanos (IBM-PSH). Trata-se da versão traduzida e adaptada por Cruz e Melo (1996) e Melo, Gomes e Cruz (1999) do Maslach Burnout Inventory-HSS (Maslach \& Jackson, 1996). O IBM-PSH é um instrumento de auto-registro com 22 itens acerca dos sentimentos relacionados com o trabalho, distribuindo-se por três dimensões: (i) exaustão emocional - analisa os sentimentos de sobrecarga emocional e exaustão devido às exigências do trabalho (e.g., "sinto-me esgotado(a) com o meu trabalho"); (ii) despersonalização pretende medir as respostas "frias", impessoais ou mesmo negativas dirigidas àqueles a quem se prestam serviços (e.g., "sinto que trato alguns clientes como se fossem objectos impessoais"); e (iii) realização pessoal - avalia os sentimentos de competência profissional e de sucesso no trabalho com pessoas (e.g., "neste emprego consegui muitas coisas que valeram a pena"). A frequência com que cada sentimento ocorre nas três áreas é avaliada numa escala tipo Likert de sete pontos, variando entre o mínimo de 0 (Nunca) e o máximo de 6 (Todos os dias).

Escala de Saúde Física (ESF). Esse instrumento destina-se a analisar a percepção dos enfermeiros acerca da 
frequência de ocorrência de problemas físicos nos últimos três meses. A ESF é uma versão traduzida e adaptada de uma escala do Occupational Stress Indicator (Cooper, Sloan \& Williams, 1988; Cunha, Cooper, Moura, Reis \& Fernandes, 1992), englobando 12 itens respondidos em um formato tipo Likert de seis pontos ( $1=$ Nunca; $6=$ Com muita frequência). O valor total da escala é calculado por meio da soma dos valores obtidos em cada um dos itens, significando valores mais elevados uma maior tendência para experimentar problemas de saúde física.

Escala de Satisfação e Realização (ESR). Esse instrumento foi desenvolvido para avaliar os níveis de satisfação e realização profissional (Gomes, Melo \& Cruz, 2000) e teve por base instrumentos similares utilizados por Boice e Myers (1987), Rodolfa e Kraft (1988), Thoresen, Miller e Krauskopf (1989). A escala é composta por cinco questões acerca da carreira e satisfação profissional, nomeadamente, (i) a vontade em optar pela mesma via de ensino se os enfermeiros tivessem uma nova oportunidade de escolher um curso superior (respostas numa escala dicotômica de "sim" e "não"); (ii) o nível de satisfação profissional; (iii) o nível de realização profissional; (iv) o desejo de abandonar o local de trabalho durante os próximos cinco anos; e (v) o desejo de abandonar a profissão durante os próximos cinco anos. Essas últimas quatro questões foram apresentadas numa escala tipo Likert de seis pontos (1=Muito Baixo; 6=Muito Alto), significando os valores mais elevados nos diferentes itens maior satisfação e realização profissional, bem como um maior desejo de abandonar o emprego e a profissão.

\section{Procedimento}

A investigação iniciou-se com o pedido de autorização aos responsáveis dos Conselhos de Administração e/ou Gestão das várias instituições de saúde, explicando-se as metas do estudo e os procedimentos a levar a cabo na coleta, tratamento e divulgação dos dados. O pedido foi apreciado pelos respectivos Comitês de Ética e, após a anuência por escrito dos mesmos, iniciamos o trabalho de campo, abordando os Chefes de Serviço de cada local de trabalho. Posteriormente, distribuiu-se o protocolo de avaliação que incluía, anexa, uma carta de apresentação, dirigida aos participantes, acerca das metas e implicações da investigação, assegurando-se o caráter voluntário da participação. No sentido de garantir a confidencialidade e anonimato dos dados recolhidos, foi fornecido a todos os inquiridos um envelope no qual introduziam o questionário preenchido, que deveria ser posteriormente devolvido, devidamente fechado. Em cada local de trabalho foi colocado uma urna devidamente selada onde foram depositados os questionários. A abertura dos envelopes contendo os questionários recebidos foi sempre da única $\mathrm{e}$ exclusiva responsabilidade do grupo de investigadores deste estudo, de forma a garantir a confidencialidade dos dados. O Questionário que englobava os instrumentos já referidos foi distribuído entre 420 enfermeiros, tendo sido recebidos e considerados como válidos para efeitos do presente estudo 286 questionários, representando uma taxa de retorno e adesão de $68,1 \%$.

\section{Resultados}

O tratamento e análise estatística dos dados foram realizados no programa SPSS (versão 15,0 para Windows) e incluiu vários procedimentos que serão explicados ao longo desta parte do trabalho.

\section{Estrutura fatorial e consistência interna dos instrumentos}

Os procedimentos de análise que descrevemos a seguir são aplicados a três dos instrumentos utilizados (questionário de estresse, inventário de burnout e escala de saúde física), não sendo incluída a escala de satisfação e realização, uma vez que os itens foram analisados separadamente pelas dimensões anteriormente descritas.

No sentido de testar a estrutura dos instrumentos referidos, realizamos uma análise fatorial dos componentes principais, sem pré-definição do número de fatores, por meio da rotação ortogonal (procedimento varimax), com normalização de Kaiser (eigenvalue igual ou superior a 1). Para a retenção dos itens em cada uma das escalas, foram fixados os seguintes critérios: (i) saturação igual ou superior a .40 de cada item no fator hipotético e apenas em um único fator; (ii) aceitação da solução fatorial final a partir do momento em que explicasse pelo menos $50 \%$ da variância total; (iii) existência de uma coerência entre a solução fatorial e os itens que constituem cada um dos fatores; e (iv) cada fator deveria ser representado, pelo menos, por dois itens (Gorsuch, 1983; Hair, Anderson, Tatham \& Black, 1995).

No que diz respeito ao questionário de estresse nos profissionais de saúde, os resultados permitiram identificar uma versão final com oito dimensões explicadoras de $63,8 \%$ de variância, num total de 40 itens assim distribuídos: (i) cometer erros e lidar com insucessos e problemas profissionais - sentimentos negativos dos profissionais relacionados com o exercício da profissão, nomeadamente, a possibilidade de cometerem erros e de terem de lidar com os problemas e expectativas dos clientes, e a falta de confiança nos conhecimentos possuídos para resolverem as exigências inerentes à sua actividade laboral (nove itens e um valor de Alpha de 0,90); (ii) ambiente de trabalho e relações profissionais - mal-estar dos profissionais relacionados com o ambiente negativo de trabalho e com os problemas de relacionamento com os colegas de trabalho e superiores hierárquicos (sete itens e um valor de Alpha de 0,82 ); (iii) excesso de trabalho e envolvimento profissional - mal-estar relacionado com a excessiva carga de trabalho a realizar e com a possibilidade da profissão exigir o máximo envolvimento dos profissionais para conseguirem cumprir as tarefas estabelecidas (seis itens e um valor de Alpha de 0,84); (iv) instabilidade profissional e na carreira - sentimentos de insegurança dos profissionais relacionados com a sua situação laboral e carreira profissional (quatro itens e um valor de Alpha de 0,80); (v) remuneração auferida - mal-estar e descontentamento relacionados com o salário recebido (quatro itens e um valor de Alpha de 0,79); (vi) falta de reconhecimento e poder - sentimentos negativos relativos ao baixo reconhecimento da profissão e às poucas oportunidades de influência do processo de tomada de decisão (três itens e um valor de Alpha de 0,75); (vii) tarefas de 
formação e elaboração de relatórios técnicos - experiências negativas dos profissionais em situações nas quais devem elaborar e conduzir trabalhos de formação e elaborar relatórios técnicos (três itens e um valor de Alpha de 0,79); e (viii) problemas familiares - dificuldades de relacionamento familiar e conciliação entre a vida profissional e familiar (quatro itens e um valor de Alpha de 0,80). Para o cálculo do valor final em cada uma das oito dimensões do QSPS, realizamos a soma do resultado de cada uma das subescalas, dividindo depois o valor encontrado pelo total de itens de cada uma delas, fazendo assim variar os resultados entre um mínimo de 0 e um máximo de 4 . Nesse sentido, valores mais elevados significam maior percepção de estresse em cada um dos domínios avaliados.

Tal como no instrumento anterior, para o IBM-PSH procedemos ao mesmo tipo de análise da estrutura fatorial, identificando-se uma versão final explicativa de $51,4 \%$ de variância total, incluindo 19 itens distribuídos da seguinte forma: exaustão emocional, com sete itens e um valor de Alpha de 0,87 ; realização pessoal, com sete itens e um valor de Alpha de 0,78; e despersonalização, com cinco itens e um valor de Alpha de 0,67. Para efeitos do presente estudo, os valores de cada subescala da versão adaptada do IBM-PSH foram calculados adicionando os valores atribuídos aos itens de cada dimensão, dividindose depois esse total pelo número de itens de cada uma delas. Assim, os resultados totais podem variar entre um mínimo de 0 e um máximo de 6 . O valor máximo reflete elevados índices de exaustão emocional, despersonalização e realização pessoal. Elevados níveis de burnout estão associados a elevados valores de exaustão emocional e despersonalização, mas também a baixos resultados na realização pessoal.

Finalmente, no que respeita à Escala de Saúde Física, a análise fatorial exploratória permitiu agrupar nove dos itens numa única faceta (tal como proposto pelos autores), explicando-se $50 \%$ de variância associada ao instrumento, com um valor de Alpha igual a 0,87. Assim sendo, o valor total de saúde física resulta do somatório dos resultados atribuídos a cada um dos itens da escala, variando entre um mínimo de nove pontos e um máximo de 54 pontos.

\section{Estatísticas descritivas e associações entre as variáveis em estudo}

Começando pelo nível global de estresse que os enfermeiros sentem geralmente no exercício da sua profissão (primeira parte do QSPS), é de realçar que cerca de 30\% dos participantes neste estudo referiram índices elevados de estresse associados à profissão. Numa análise mais detalhada dos possíveis problemas que podem contribuir para essa situação, a Tabela 1 ilustra as 10 fontes de estresse incluídas na segunda parte do QSPS que foram consideradas como geradoras de maior pressão, apresentando-se nessa análise os valores iguais ou superiores a três em cada um dos 70 itens. De modo geral, são as questões relacionadas com as implicações dos erros cometidos (e.g., "tomar decisões onde os erros podem ter consequências graves"), as relações profissionais (e.g., "a incompetência e/ou inflexibilidade dos meus superiores hierárquicos"), a relação com os clientes (e.g., "comunicar aos clientes decisões e/ou informações difíceis ou delicadas"), as apresentações do trabalho aos outros (e.g., "falar ou fazer apresentações em público"), a carreira profissional (e.g., "a falta de perspectivas de desenvolvimento e promoção na carreira") e o excesso de trabalho e gestão do tempo (e.g., "falta de tempo para realizar adequadamente as minhas tarefas profissionais") que mais parecem perturbar os participantes nesta investigação

Essa variedade de problemas profissionais representa, em grande medida, os dados relativos às dimensões de estresse da escala, os quais são apresentados na Tabela 2. Assim, e considerando uma vez mais os resultados extremos da escala Likert (valores 3 e 4), constata-se que os trabalhos de formação e elaboração de relatórios técnicos, o cometer erros e lidar com os insucessos, o excesso de trabalho, a instabilidade profissional e o mau ambiente de trabalho são as áreas de estresse que englobam, em grande medida, as 10 principais fontes de estresse relatadas pelos enfermeiros.

Relativamente aos níveis de esgotamento dos enfermeiros, utilizamos as indicações sugeridas por Shirom (1989) para calcular os valores problemáticos em cada uma das três facetas do burnout. Nesse caso, o autor propõe a utilização dos valores da escala Likert como referência para estabelecer os "pontos" de corte das três escalas. Assim sendo, são indicados dois critérios de cálculo alternativos, um menos restritivo (valor igual ou superior à frequência "algumas

Tabela 1. Ranking dos aspectos sentidos pelos enfermeiros como geradores de "bastante" ou "muito" estresse (*).

\begin{tabular}{|c|c|c|}
\hline Item - Fonte de estresse & $\mathrm{n}$ & $\%$ \\
\hline $\begin{array}{l}\text { Tomar decisões onde os erros podem ter } \\
\text { consequências graves }\end{array}$ & 196 & 68,6 \\
\hline $\begin{array}{l}\text { A incompetência e/ou inflexibilidade } \\
\text { dos meus superiores hierárquicos }\end{array}$ & 174 & 60,4 \\
\hline $\begin{array}{l}\text { Comunicar aos clientes decisões e/ou } \\
\text { informações "difíceis" ou "delicadas" }\end{array}$ & 171 & 59,8 \\
\hline $\begin{array}{l}\text { As implicações e consequências dos in- } \\
\text { sucessos ou erros que eu possa cometer }\end{array}$ & 169 & 59,1 \\
\hline Falar ou fazer apresentações em público & 165 & 57,7 \\
\hline $\begin{array}{l}\text { A falta de perspectivas de desenvolvi- } \\
\text { mento e promoção na carreira }\end{array}$ & 165 & 57,7 \\
\hline $\begin{array}{l}\text { Falta de tempo para realizar adequada- } \\
\text { mente as minhas tarefas profissionais }\end{array}$ & 159 & 55,6 \\
\hline $\begin{array}{l}\text { Assumir responsabilidades não relacio- } \\
\text { nadas com a formação que recebi }\end{array}$ & 159 & 55,6 \\
\hline $\begin{array}{l}\text { O favoritismo e/ou discriminação "enco- } \\
\text { bertos" no meu local de trabalho }\end{array}$ & 157 & 54,9 \\
\hline $\begin{array}{l}\text { Sentir que não há nada a fazer para } \\
\text { resolver os problemas dos meus clientes }\end{array}$ & 153 & 53,5 \\
\hline
\end{tabular}


Tabela 2. Ranking das dimensões de estresse sentidas pelos enfermeiros como geradoras de "bastante" ou "muito" estresse (*).

\begin{tabular}{|c|c|c|}
\hline Dimensão de estresse & $\mathrm{n}$ & $\%$ \\
\hline $\begin{array}{l}\text { Trabalho de formação e elaboração de } \\
\text { relatórios técnicos }\end{array}$ & 283 & 25,4 \\
\hline $\begin{array}{l}\text { Cometer erros e lidar com insucessos e } \\
\text { problemas profissionais }\end{array}$ & 285 & 19,3 \\
\hline $\begin{array}{l}\text { Excesso de trabalho e envolvimento } \\
\text { profissional }\end{array}$ & 285 & 18,6 \\
\hline Instabilidade profissional e na carreira & 283 & 17,7 \\
\hline $\begin{array}{l}\text { Ambiente de trabalho e relações profis- } \\
\text { sionais }\end{array}$ & 285 & 15,1 \\
\hline Remuneração auferida & 284 & 13,7 \\
\hline Falta de reconhecimento e poder & 281 & 10,7 \\
\hline Problemas familiares & 284 & 7,4 \\
\hline
\end{tabular}

vezes por mês" na escala Likert) e outro mais conservador (valor igual ou superior à frequência "algumas vezes por semana" na escala Likert). No nosso caso, procuramos usar um critério intermediário que conciliasse os dois descritos, assumindo-se como valor de corte a frequência "uma vez por semana" da escala Likert para as dimensões de exaustão emocional e cinismo, e "uma vez por mês" para a dimensão de eficácia profissional (recordamos que nesta última dimensão a leitura dos resultados deve ser interpretada em sentido inverso, ou seja, valores menos elevados na escala significam menor eficácia profissional). Os resultados obtidos apontaram um valor assinalável de profissionais com exaustão emocional $(15,5 \%)$, seguindo-se $4 \%$ com despersonalização e menos de $1 \%$ com problemas de realização pessoal.

No que concerne aos índices de saúde física, os resultados obtidos sugerem que "sentir-se extremamente cansado(a) ou exausto(a)", "ter a sensação de não querer levantar-se de manhã", "dificuldades em adormecer ou em manter um sono contínuo", "tendência para comer, beber ou fumar mais do que é habitual" e "enxaquecas e dores de cabeça" representam os indicadores de saúde experimentados mais frequentemente e referidos por cerca de 13 a 35\% dos profissionais.

Quanto aos indicadores de satisfação e realização profissional, alguns dados merecem a nossa atenção. Em primeiro lugar, deve-se assinalar que praticamente $1 / 4$ dos participantes (24\%) não voltaria a escolher o mesmo curso e, consequentemente, a mesma profissão se tivessem uma nova oportunidade de optar por uma saída profissional. Em termos da satisfação profissional, $9 \%$ estão bastante insatisfeitos com a sua profissão, enquanto $28 \%$ estão bastante satisfeitos com a sua profissão. Nesse mesmo sentido, os valores de realização profissional são bastante baixos para $2 \%$ dos enfermeiros e bastante altos para $24 \%$ (a grande maioria sente-se moderadamente realizada). Já no que diz respeito ao desejo de abandonar o local de trabalho e a profissão, os valores extremaram-se ainda mais, colocando o grupo dos que tencionam deixar o lugar onde exercem funções com $12 \%$ e dos que pretendem deixar a profissão com $8 \%$, contrastando com os $68 \%$ que manifestaram a vontade em continuar no mesmo local de exercício de funções e os $78 \%$ que pretendem manter-se no mesmo contexto profissional.

Por último, as análises das associações e correlações existentes entre todas as variáveis em estudo foram realizadas por meio do cálculo dos coeficientes de correlação de Pearson. Esses coeficientes encontram-se na Tabela 3. Aumentos nos níveis globais de estresse aparecem associados, por um lado, a valores superiores de exaustão emocional e, por outro lado, a experiências profissionais mais negativas nas oito áreas de estresse do QSPS.

De igual modo, constata-se uma relação entre as oito dimensões que compõem o questionário de estresse, sendo positivo o sentido da associação, o que equivale a dizer que aumentos em cada um dos oito domínios implica igualmente aumento nas restantes sete facetas. Quanto às associações entre as oito áreas de estresse e as outras medidas em estudo, é de salientar que os problemas de saúde física e os níveis de exaustão emocional do IBM-PSH tenderem a ser mais elevados com aumentos em qualquer uma das dimensões de estresse. Já com um menor número de associações, deve ser salientado que a despersonalização do IBM-PSH aumenta com as queixas acerca do excesso de trabalho, do mau ambiente de trabalho, da falta de reconhecimento, da remuneração auferida e dos problemas familiares. Com apenas uma associação em causa, devemos assinalar que os problemas de estresse relacionados com os trabalhos de formação e elaboração de relatórios técnicos traduzem-se por um menor sentimento de realização pessoal do IBM-PSH. No que respeita à relação entre o estresse e a satisfação/ realização, é evidente a tendência da satisfação profissional diminuir à medida que os profissionais experimentam mais problemas relacionados com o cometer erros, o excesso de trabalho, a instabilidade profissional e a remuneração auferida. Já os sentimentos de realização profissional parecem mais condicionados pelos problemas da instabilidade profissional e do salário recebido. Nesse mesmo sentido, o desejo de abandonar o local de emprego aparece associado ao descontentamento com o salário.

No que se refere aos problemas de saúde física, para além das relações já referidas com as dimensões de estresse, deve ainda ser apontada a existência de uma associação positiva com a despersonalização, o desejo de abandonar o emprego e a profissão e uma associação negativa com a satisfação profissional.

Em termos do burnout, a escala com efeitos negativos mais generalizados é a exaustão emocional que, para além de implicar aumentos ao nível da despersonalização, significa uma menor satisfação e realização profissional, em paralelo, como um maior desejo de abandonar o local de emprego e a profissão. Já a maior realização pessoal associa-se a uma menor despersonalização e a uma maior satisfação e realização profissional. No caso da despersonalização, os seus efeitos fizeram-se sentir num menor sentimento de realização profissional.

Por último, para as correlações entre as dimensões da satisfação e realização, verificou-se a relação esperada, 


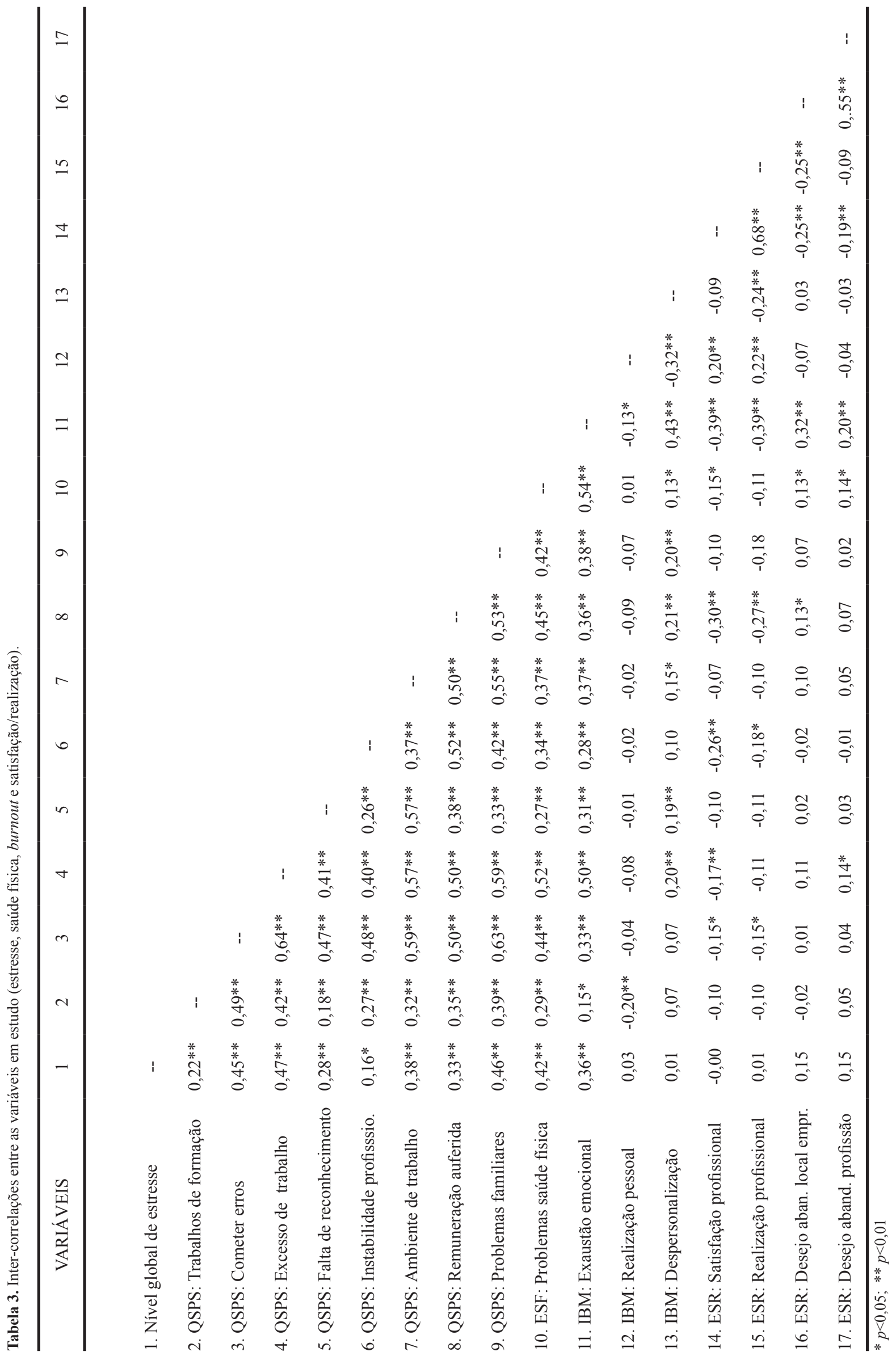


ou seja, a satisfação correlaciona-se positivamente com a realização profissional e negativamente com o desejo de abandonar o local de emprego e a profissão. De igual modo, a maior realização profissional significou um menor desejo de deixar o local de trabalho e esta última dimensão associou-se positivamente com o desejo de abandonar a profissão.

\section{Variáveis preditoras da experiência de burnout, saúde física, satisfação e realização profissional}

Nestas análises procurou-se compreender quais as dimensões que melhor poderiam explicar a experiência de burnout, saúde física, satisfação e realização profissional. Nesse sentido, realizamos análises de regressão múltipla, por meio do método stepwise. Os resultados dessa análise são apresentados na Tabela 4.

Assim sendo, começamos por estudar o impacto das oito áreas de estresse do QSPS nas três dimensões do IBMPSH. No caso da exaustão emocional, o modelo final ficou representado por duas variáveis de estresse, o excesso de trabalho e envolvimento profissional e as queixas acerca da remuneração auferida, que no seu conjunto explicaram $26 \%$ da variância. Esse valor de variância baixou para a realização pessoal, que viu apenas $4 \%$ da sua variância explicada pelos trabalhos de formação e elaboração de relatórios técnicos. Já no que respeita à despersonalização, os problemas familiares, a falta de reconhecimento e poder e o cometer erros explicaram $7 \%$ da variância.
De seguida, incluímos como variáveis preditoras as fontes de estresse e as dimensões de burnout para analisar o impacto ao nível dos problemas de saúde física, da satisfação e da realização profissional. Desse modo, e começando pela ESF, verificamos que a exaustão emocional, o excesso de trabalho, os problemas com a remuneração auferida e a despersonalização entraram na análise final, explicando $40 \%$ da variância associada aos problemas de saúde física. No que concerne à satisfação profissional, as três dimensões do IBM-PSH, bem como os problemas com a remuneração e o ambiente de trabalho do QSPS, explicaram 25\% da variância. Por fim, para a realização profissional, 22\% da sua variância foi igualmente explicada pelas dimensões da exaustão emocional e realização pessoal do IBM-PSH, bem como pelos problemas relacionados com a remuneração e o excesso de trabalho do QSPS.

\section{Diferenças na experiência de estresse, burnout, saúde física, satisfação e realização profissional}

Nessa etapa do estudo, procuramos observar eventuais diferenças nas dimensões analisadas pelos instrumentos de avaliação (variáveis dependentes) em função de algumas características demográficas e profissionais da nossa amostra (variáveis independentes). Para tal, foram constituídos os seguintes grupos de comparação: sexo, idade, experiência profissional, situação contratual, trabalho por turnos e número de horas de trabalho por semana. Foi aplicado, consoante os

Tabela 4. Sumário das análises de regressão múltipla.

\begin{tabular}{|c|c|c|c|c|c|c|}
\hline VARIÁVEIS OBSERVADAS & VARIÁVEIS PREDITORAS & $R^{2}$ & $R^{2 a j u s t .}$ & Beta & $t$ & $F$ \\
\hline \multirow[t]{2}{*}{ IBM: Exaustão emocional } & Excesso de trabalho & 0,244 & 0,241 & 0,423 & 7,08 & $88,08 * * *$ \\
\hline & Remuneração & 0,260 & 0,255 & 0,146 & 2,45 & $47,86 * * *$ \\
\hline IBM: Realização pessoal & Trabalhos de formação & 0,045 & 0,041 & $-0,212$ & $-3,58$ & $12,80 * * *$ \\
\hline \multirow[t]{3}{*}{ IBM: Despersonalização } & Problemas familiares & 0,042 & 0,039 & 0,260 & 3,44 & $12,05 * *$ \\
\hline & Falta de reconhecimento & 0,059 & 0,052 & 0,193 & 2,91 & $8,52 * * *$ \\
\hline & Cometer erros & 0,078 & 0,067 & $-0,190$ & $-2,35$ & $7,61 * * *$ \\
\hline \multirow[t]{4}{*}{ ESF: Problemas de Saúde Física } & Exaustão emocional & 0,274 & 0,271 & 0,392 & 6,64 & $102,64 * * *$ \\
\hline & Excesso de trabalho & 0,361 & 0,356 & 0,253 & 4,31 & $76,41 * * *$ \\
\hline & Remuneração & 0,387 & 0,380 & 0,197 & 3,61 & $56,76^{* * *}$ \\
\hline & Despersonalização & 0,404 & 0,395 & $-0,146$ & $-2,81$ & $45,63 * * *$ \\
\hline \multirow[t]{5}{*}{ ESR: Satisfação profissional } & Exaustão emocional & 0,156 & 0,153 & $-0,425$ & $-6,73$ & $49,66 * * *$ \\
\hline & Remuneração & 0,189 & 0,183 & $-0,267$ & $-4,33$ & $31,17 * * *$ \\
\hline & Realização pessoal & 0,216 & 0,207 & 0,205 & 3,69 & $24,53 * * *$ \\
\hline & Despersonalização & 0,241 & 0,229 & 0,186 & 3,06 & $21,07 * * *$ \\
\hline & Ambiente de trabalho & 0,264 & 0,250 & 0,183 & 2,91 & $19,03 * * *$ \\
\hline \multirow[t]{4}{*}{ ESR: Realização profissional } & Exaustão emocional & 0,150 & 0,146 & $-0,373$ & $-4,94$ & $32,06 * * *$ \\
\hline & Realização pessoal & 0,189 & 0,180 & 0,178 & 2,70 & $20,97 * * *$ \\
\hline & Remuneração & 0,214 & 0,200 & $-0,245$ & $-3,14$ & $16,21 * * *$ \\
\hline & Excesso de trabalho & 0,236 & 0,219 & 0,188 & 2,28 & $13,74 * * *$ \\
\hline
\end{tabular}

$* * * p<0,001 ; * * p<0,01$ 
casos em questão, análises de variância One-way (ANOVA), seguidas de comparações post-hoc com o teste de Scheffé e teste $t$ para amostras independentes.

No que concerne à distinção em função do sexo, verificamos seis dimensões de estresse com diferenças significativas, resultando em qualquer um dos casos em uma maior experiência de estresse por parte das mulheres. Mais concretamente, as divergências foram visíveis nas seguintes áreas: (i) cometer erros e lidar com os insucessos profissionais $(t=-5,13 ; p<0,001)$ (valor médio das mulheres foi de 2,52 e dos homens, 1,98); (ii) instabilidade profissional e na carreira ( $t=-2,76 ; p<0,01)$ (valor médio das mulheres foi de 2,22 e dos homens, 1,83); (iii) excesso de trabalho e envolvimento profissional $(t=-3,45 ; p<0,01)$ (valor médio das mulheres foi de 2,37 e dos homens, 1,99); (iv) remuneração auferida $(t=-2,66 ; p<0,01)$ (valor médio das mulheres foi de 2,07 e dos homens, 1,71); (v) falta de reconhecimento e poder $(t=-2,63 ; p<0,01)$ (valor médio das mulheres foi de 2,27 e dos homens, 1,95); e (vi) trabalhos de formação e relatórios técnicos $(t=-3,22 ; p<0,01)$ (valor médio das mulheres foi de 2,57 e dos homens, 2,12). Com relação à saúde física, as mulheres $(\mathrm{M}=3,15)$ apresentaram mais problemas do que os homens $(\mathrm{M}=2,50)(t=-4,49 ; p<0,001)$ e, com um padrão inverso, estes últimos evidenciaram uma maior despersonalização $(\mathrm{M}=1,06)$ do que as suas colegas do sexo feminino $(\mathrm{M}=0,77)(t=2,44 ; p<0,05)$.

Em seguida, testamos a existência de discrepâncias em função da idade dos enfermeiros, constituindo-se três grupos distintos que indicassem as várias etapas do desenvolvimento profissional (até 30 anos, dos 31 aos 50 anos e mais de 50 anos de idade). Começando pelas dimensões de estresse, há que destacar duas diferenças. Na instabilidade profissional e na carreira $\left(F_{(2,218)}=17,61 ; p<0,001\right)$, constatou-se pelo post-hoc de Scheffé que os profissionais mais novos (até 30 anos) apresentaram mais problemas $(\mathrm{M}=2,70)$ do que os seus colegas com idades intermediárias $(\mathrm{M}=1,98)$ e mais velhos $(\mathrm{M}=1,78)$. Nesse mesmo sentido, os problemas relacionados com a remuneração auferida $\left(F_{(2,219)}=5,05 ; p<0,01\right)$ tendem, de acordo com o post-hoc, a perturbar mais os enfermeiros com idades até 30 anos $(\mathrm{M}=2,31)$ do que os seus colegas com idades entre os 31 e os 50 anos $(\mathrm{M}=1,93)$. Para as dimensões do IBM-PSH, devem ser acentuadas duas diferenças estatisticamente significativas. Nesse caso, na dimensão da realização pessoal $\left(F_{(2,218)=} 3,33 ; p<0,05\right)$ destacaram-se, segundo o post-hoc, as diferenças entre o grupo de participantes com idades compreendidas entre os 31 e os 50 anos $(M=4,41)$ e os com mais de 50 anos $(M=5,14)$, assumindo este último grupo maiores níveis de realização do ponto de vista pessoal. A segunda diferença que deve ser assinalada prende-se com a despersonalização $\left(F_{(2,218)}=7,61 ; p<0,01\right)$, verificando-se, de acordo com o post-hoc, que os enfermeiros com idades até 30 anos assumiram uma maior tendência para a despersonalização $(\mathrm{M}=0,99)$ do que os seus colegas com idades intermediárias $(\mathrm{M}=0,67)$ e mais velhos $(\mathrm{M}=0,27)$. No último conjunto de comparações (variáveis de satisfação e realização), observaram-se três diferenças estatisticamente significativas entre os três grupos de idades. Assim, o nível de realização profissional $\left(F_{(2,185)}=4,60 ; p<0,05\right)$ colocou, de acordo com o post-hoc, o grupo dos mais velhos (mais de 50 anos) com maiores níveis de realização $(\mathrm{M}=5,00)$ do que os grupos com idades intermediárias $(\mathrm{M}=4,07)$ e mais baixas $(\mathrm{M}=3,96)$. Já a vontade em abandonar o local de emprego $\left(F_{(2,215)}=5,74 ; p<0,01\right)$ contrastou, de acordo com o post-hoc, o grupo mais velho $(\mathrm{M}=3,58)$ com os outros dois grupos, nomeadamente, o grupo dos 31 aos 50 anos $(\mathrm{M}=2,03)$ e o grupo dos 30 anos $(M=2,31)$. Nesse mesmo sentido, foram os mais velhos $(\mathrm{M}=3,83)$ que evidenciaram um maior desejo de abandonar a profissão $\left(F_{(2,216)}=15,42 ; p<0,001\right)$ quando comparados com os seus colegas com idades entre os 31 e os 50 anos $(M=1,75)$ e os com até 30 anos $(M=1,58)$.

O terceiro conjunto de análises comparou três grupos de participantes com diferentes anos de trabalho, procurandose discriminar entre enfermeiros em início de carreira (até 5 anos), com experiência intermediária de trabalho (6 a 15 anos) e com bastante prática nas suas funções (mais de 15 anos). Os resultados evidenciaram quatro diferenças com significado estatístico. Em primeiro lugar, o estresse relacionado com a instabilidade profissional e na carreira $\left(F_{(2,254)}=7,32\right.$; $p<0,01$ ), tendo-se verificado, de acordo com o post-hoc, que os profissionais com até cinco anos de trabalho eram mais sensíveis a esse problema $(M=2,40)$ do que seus colegas com mais de 15 anos de trabalho $(M=1,75)$. De um modo ainda mais extensivo, as dificuldades relacionadas com a remuneração auferida $\left(F_{(2,255)}=4,84 ; p<0,01\right)$ suscitaram menos estresse nos profissionais com mais de 15 anos de trabalho $(\mathrm{M}=1,62)$ relativamente aos seus colegas com até 5 anos $(\mathrm{M}=2,05)$ e entre 6 a 15 anos de prática profissional $(M=2,12)$. Em outro sentido, mas em uma lógica de resultados congruentes a esses, constatou-se que foram os mais velhos $(\mathrm{M}=2,49)$ que manifestaram maior desejo de abandonar a profissão $\left(F_{(2,}\right.$ $\left.{ }_{249)}=10,19 ; p<0,001\right)$ quando comparados com os enfermeiros com experiência intermediária $(M=1,49)$ e em início de carreira $(\mathrm{M}=1,63)$. A última diferença a salientar prende-se com a tendência para a despersonalização $\left(F_{(2,253)}=3,25\right.$; $p<0, .05)$, constando-se, segundo o teste de post-hoc, que os menos experientes (até 5 anos) tendiam a apresentar mais problemas nessa área $(\mathrm{M}=0,95)$ do que os mais experientes (mais de 15 anos) $(\mathrm{M}=0,58)$.

A comparação entre profissionais com situações mais estáveis na carreira e profissionais com contratos a prazo/ termo representou a quarta categoria de comparação. Nesse caso, e tal como seria de esperar, foi o mal-estar associado à instabilidade profissional e na carreira que diferenciou os grupos $(t=-4,42 ; p<0,001)$, colocando os enfermeiros em situação precária a sentir mais estresse $(M=2,79)$ do que os seus colegas com vínculos laborais estáveis $(\mathrm{M}=2,14)$.

O quinto agrupamento diz respeito à realização de trabalho por turnos, realizando-se uma comparação entre profissionais com horários "normais" de trabalho (diurnos) e os que fazem trabalho por turnos com diferentes horários de serviço (incluindo durante a noite). Nesse caso, o estresse associado à instabilidade profissional e à carreira $(t=-4,10$; $p<0,001)$ tende a influenciar mais o grupo que realiza trabalho por turnos $(M=2,42)$ relativamente aos seus colegas em horário habitual $(\mathrm{M}=1,75)$. Com relação às dimensões do IBM-PSH, a tendência para despersonalizar $(t=3,47 ; \mathrm{p}<0,01)$ foi superior nos que faziam turnos $(\mathrm{M}=0,81)$ em comparação com seus colegas que não faziam $(\mathrm{M}=044)$. Também a realização profissional da ESR revelou resultados significativos $(t=-2.76 ; p<0,01)$, uma vez que o grupo que não realizava 
turnos $(M=4,49)$ evidenciou mais sentimentos de realização do que os seus colegas $(\mathrm{M}=3,99)$.

No último conjunto de comparações, colocou-se sob análise diferentes horas de trabalho por semana (até 35 horas e mais de 35 horas). Nesse caso, verificaram-se duas diferenças significativas nas dimensões do IBM-PSH, nomeadamente na exaustão emocional $(t=-2,05 ; p<0,05)$, sendo o grupo que faz mais horas de trabalho $(\mathrm{M}=2,09)$ o que revelou mais problemas comparativamente aos que realizavam menos horas $(\mathrm{M}=1,78)$, e na tendência para despersonalizar $(t=-$ $4,09 ; p<0,001)$, sendo também superior no grupo com mais horas de trabalho $(\mathrm{M}=1,12)$ relativamente ao com menos horas $(\mathrm{M}=0,67)$. Quanto ao desejo de abandonar o local de emprego $(t=2,34 ; p<0,05)$, foi o grupo com até 35 horas de trabalho que manifestou mais esta vontade $(M=2,32)$ quando comparado com os que ultrapassaram esse número de horas $(\mathrm{M}=1,89)$. A vontade em deixar a profissão apresentou resultados congruentes com os anteriores $(t=3,24 ; p<0,01)$, uma vez que é o grupo que trabalhava até 35 horas $(M=1,92)$ que descreveu maior desejo de abandonar a profissão em relação ao que foi descrito pelos seus colegas com mais horas de trabalho $(\mathrm{M}=1,42)$.

\section{Discussão}

O estudo do estresse ocupacional em profissionais de saúde tem suscitado a atenção dos investigadores que procuram compreender esse problema em termos das manifestações e consequências no funcionamento dos trabalhadores da área da saúde, bem como no tipo de serviços que prestam aos outros. A investigação levada a cabo insere-se nessa lógica, fornecendo indicações acerca da experiência de trabalho numa amostra de enfermeiros portugueses e, adicionalmente, procurando contribuir para o desenvolvimento de metodologias de avaliação específicas para esses profissionais.

Começando pelo último aspecto, devemos salientar os dados obtidos no Questionário de Estresse nos Profissionais de Saúde (QSPS), uma vez que as metodologias de avaliação dirigidas a essas populações são ainda escassas. Assim, de um modo geral, as estruturas fatoriais dos instrumentos confirmaram as dimensões esperadas, particularmente no Inventário de Burnout e na Escala de Saúde Física. No que se refere ao QSPS, este apresentou oito dimensões, que permitem um entendimento bastante lato das áreas de estresse no trabalho dos enfermeiros. Por outro lado, os valores de alpha encontrados foram bastante aceitáveis, situando-se acima do ponto crítico de 0,70 , considerado como indicador de uma boa consistência interna (Nunnally, 1978). Adicionalmente, as correlações entre as várias medidas utilizadas apresentaram o "comportamento" esperado, uma vez que aumentos em qualquer uma das dimensões de estresse implicaram resultados mais elevados nas restantes. De igual modo, maior estresse associou-se a mais problemas de saúde física e a maior exaustão emocional. Deve ainda ser salientado que não foram verificados problemas de "redundância" entre as variáveis em estudo, não tendo nenhuma das correlações atingido o ponto crítico de 0,70 , sugerido por Pedhazur (1982) como suscitando problemas de multicolinearidade. Uma vez mais, esses dados são particularmente importantes no questionário de estresse, pois este estudo representou a primeira validação do instrumento, dando assim indicações positivas acerca da relevância e interesse na aplicação aos profissionais de saúde.

No que se refere à observação dos valores médios e frequências das várias dimensões em análise, há que se realçar alguns aspectos. Desde logo, a situação de 30\% dos enfermeiros experimentarem níveis elevados de estresse justifica a importância do estudo do tema junto dessa classe profissional, bem como a necessidade de se desenvolverem estratégias de intervenção que possam ajudar na gestão da pressão inerente a essa profissão. Por outro lado, os valores observados encontram paralelo em outros estudos realizados em Portugal, seja com psicólogos (Gomes, Melo \& Cruz, 2000), seja com professores (Cruz \& Freitas, 1988, Junho; Cruz \& Mesquita, 1988, Setembro; Gomes, Silva, Mourisco, Mota \& Montenegro, 2006; Pinto, 2000), tendo sido obtidos valores médios de estresse elevados, acima dos $40 \%$. No entanto, os resultados verificados neste estudo situam-se abaixo dos valores encontrados por Petterson e cols. (1995), que descreveram cerca de $80 \%$ de problemas de estresse em uma amostra de enfermeiros suecos. Analisando mais detalhadamente as dimensões de estresse que poderão estar na origem desse problema, os resultados por nós encontrados sugerem as dificuldades em realizar trabalhos de formação e elaborar relatórios técnicos, a possibilidade de cometer erros, o excesso de trabalho e a instabilidade profissional como perturbando particularmente os enfermeiros. Esses indicadores encontram correspondência nos dados de outras investigações sobre fontes de pressão em profissionais de saúde, principalmente no que diz respeito à sobrecarga de trabalho, às pressões de tempo e aos aspectos relacionados com a carreira e com a realização profissional (Douglas, Meleis, Eribes \& Kim, 1996; Melo, Gomes \& Cruz, 1997; Narayan, Menon \& Spector, 1999; Payne \& Firth-Cozens, 1987).

Quanto aos níveis globais de burnout, vale a pena mencionar os índices acentuados de exaustão emocional (mais de $15 \%$ ) evidenciada pelos enfermeiros, sendo esta a dimensão mais problemática da experiência de esgotamento. Resultados semelhantes foram encontrados por Queirós (2005) num estudo com enfermeiros portugueses, sendo a dimensão da exaustão emocional a mais elevada $(27 \%)$ das três áreas de burnout, seguida do cinismo com 16\% (dimensão semelhante à despersonalização do IBM-PSH) e da ineficácia profissional (2\%). Também no nosso caso, observamos diminuições substanciais nas restantes dimensões do IBM-PSH, nomeadamente na despersonalização (4\%), o que é particularmente positivo nesse tipo de profissionais, e na realização pessoal (1\%). Dito por outras palavras, a possibilidade de esgotamento nos enfermeiros participantes nesta investigação está fundamentalmente associada a sensações de cansaço e exaustão emocional, mas tais resultados não parecem condicionar o tipo de serviços prestados aos pacientes nem tão pouco os sentimentos de eficácia e realização pessoal. Aliás, o resultado da realização pessoal é corroborado pelos valores obtidos na escala de satisfação e realização profissional, uma vez que os valores em ambas as subescalas variam entre $9 \%$ de grande insatisfação e $2 \%$ de falta de realização. No entanto, não devemos descurar a situação de praticamente $25 \%$ dos participantes não voltarem a escolher a sua profissão 
se tivessem uma nova oportunidade de optar por uma saída profissional. Curioso é ainda o resultado de alguns dos indicadores de saúde física estarem mais associados à dimensão da exaustão emocional do IBM-PSH, uma vez que o "sentir-se extremamente cansado(a) ou exausto(a)", o "ter a sensação de não querer levantar-se de manhã", e as "dificuldades em adormecer ou em manter um sono contínuo" representaram os problemas de saúde física mais referenciados e que, de algum modo, encontram correspondência nas características identificadoras da exaustão emocional ("sensações de cansaço de manhã", "sentimentos de esgotamento com o trabalho", "sentir-se no limite das capacidades").

A questão que se pode colocar a seguir prende-se com a contribuição das dimensões de estresse na explicação da experiência de esgotamento, saúde física, satisfação e realização profissional. Os dados das análises de regressão revelaram alguns aspectos interessantes. Assim, é curioso verificar que é precisamente a dimensão de exaustão emocional que tem o maior valor de variância explicada pelos factores do QSPS (26\%), sendo essa a faceta mais prevalente das três subescalas que constituem o IBM-PSH. De algum modo, as análises de regressão demonstraram que é o excesso de trabalho (que implica um maior ritmo de vida e maior cansaço) e o descontentamento com a remuneração auferida que melhor explicam as sensações de cansaço e exaustão emocional. Tais resultados encontram paralelo em um trabalho realizado com enfermeiros holandeses, constatando-se igualmente uma grande importância do excesso de trabalho na explicação da exaustão emocional (Tummers, Merode, Landeweerd \& Candel, 2003). Já os problemas de saúde física são fundamentalmente explicados pela exaustão emocional (o que comprova a importância da análise conjunta dessas duas dimensões), o excesso de trabalho, o descontentamento com a remuneração e a tendência para uma maior despersonalização. No que se refere às dimensões de satisfação e realização profissional, devemos destacar a importância da exaustão emocional e da realização pessoal do IBM-PSH bem como da insatisfação com a remuneração auferida. No seu conjunto, os dados obtidos sugerem que é a dimensão mais "física" do esgotamento (exaustão emocional), a mais bem explicada pelas dimensões de estresse.

No último conjunto de análises realizadas, consideramos seis grupos de comparação na amostra deste estudo. Em primeiro lugar, as diferenças de sexo colocaram as mulheres com mais problemas de estresse em seis dos oito fatores avaliados pelo QSPS e com mais dificuldades de saúde física que os seus pares. Tais resultados vão de encontro à literatura nesse domínio, que tende de um modo geral a atribuir às mulheres níveis mais elevados de estresse relacionado com vários aspectos do trabalho (Calais, Andrade \& Lipp, 2003; Cooper, Dewe \& O`Driscoll, 2001; Cruz \& Mesquita, 1988, Setembro; Gomes \& Cruz, 2004; Melo, Gomes \& Cruz, 1997; Pinto, 2000). No entanto, e de forma inversa, as mulheres tendem a despersonalizar menos que os homens mas, uma vez mais, esse resultado está de acordo com dados de outros estudos. A título ilustrativo, Gil-Monte (2002), em um estudo com profissionais espanhóis de enfermagem, constatou que os homens tendiam a evidenciar maiores níveis de despersonalização do que as mulheres.
Já no que respeita à importância da idade e da experiência profissional, em termos gerais, deve-se salientar que os enfermeiros mais novos evidenciarem, como seria de esperar, mais problemas relacionados com a instabilidade profissional e o salário auferido, mas também uma maior tendência para a despersonalização. Nesse último caso, pode-se levantar a hipótese da despersonalização representar uma estratégia de gestão da inexperiência do profissional face aos seus pacientes, "afastando-se" emocionalmente dos problemas que apresentam. Por outro lado, e como seria igualmente esperado, foram os mais velhos que manifestaram maior desejo de abandonar o emprego e a profissão, embora representassem um grupo mais realizado do ponto de vista pessoal e profissional. Para tal, muito poderá contribuir a estabilidade profissional que alcançam na etapa final da carreira laboral. Esses dados são corroborados pela situação contratual, uma vez que aqueles que possuem vínculos de trabalho precários nos respectivos locais de trabalho assumem mais estresse associado à instabilidade profissional e à carreira. De um modo geral, esse padrão de resultados corrobora as indicações mais gerais da investigação, na qual se tem vindo a salientar a possibilidade de existir uma relação entre a idade e a tendência para o esgotamento (Leiter \& Harvie, 1996), salientando-se a ideia dos profissionais de serviços humanos com menor experiência poderem ser mais vulneráveis ao burnout (Cavalheiro, Moura Junior \& Lopes, 2008; Queirós, 2005).

A diferenciação dos profissionais em função da realização de trabalho por turnos chama-nos a atenção para os potenciais efeitos negativos dessa organização de trabalho, pois os enfermeiros que se encontram a trabalhar desse modo sentiram mais problemas de esgotamento (ao nível da despersonalização), maior mal-estar relacionado com a instabilidade profissional e menor realização profissional. Tais resultados vão de encontro a outros dados da investigação, que realçam a possibilidade do trabalho por turnos não ser imune a "efeitos secundários" para aqueles que são expostos a essa organização laboral (Parikh, Taukari \& Bhattacharya, 2004; Queirós, 2005). Além disso, não devemos igualmente descurar as indicações da literatura que demonstram que o trabalho em sistema de rotação de horários (como acontece frequentemente em Portugal) é precisamente a metodologia que piores consequências traz para o profissional, não só em termos do seu rendimento, mas também em relação à satisfação, ao número de erros cometidos, às lesões e aos acidentes no trabalho e ao número de dias de ausência ao trabalho por motivos de doença (Coffey, Skipper \& Jung, 1988; Effinger, Nelson \& Starr, 1995).

Finalmente, o maior número de horas de trabalho por semana também correspondeu a níveis mais acentuados de exaustão emocional e despersonalização por parte dos enfermeiros (podendo-se questionar quais os efeitos que isso poderá ter sobre a qualidade do trabalho prestado) e significaram menor desejo em abandonar o emprego e a profissão (podendo aqui existir uma relação entre ser mais novo e realizar mais horas de trabalho semanal). Esses resultados encontram correspondência noutro estudo realizado em Portugal com enfermeiros, tendo-se verificado que os profissionais em horários de trabalho acrescidos (42 horas semanais) 
evidenciaram maiores índices de exaustão emocional e física, de cinismo e de burnout conjugal (Queirós, 2005).

Em síntese, os dados obtidos neste estudo reforçam a importância da investigação acerca do estresse ocupacional nessa classe profissional (e noutras, como os médicos), uma vez que a elevada experiência de mal-estar e pressão profissional influenciam uma significativa quantidade de trabalhadores, podendo colocar-se a questão de saber quais as condições reais em que exercem as suas funções. Paralelamente, observou-se que a experiência de estresse é multifacetada, existindo uma amplitude razoável de dimensões que podem contribuir para os problemas laborais dos enfermeiros. Esse aspecto tem como consequência a necessidade das futuras propostas de intervenção deverem ser dirigidas ao "combate" dos vários problemas laborais e incluírem estratégias de intervenção variadas, uma vez que é totalmente diferente delinear uma intervenção dirigida à melhoria da relação com os pacientes e outra que ajude os profissionais a gerirem melhor o estresse associado ao "cometer erros" ou ao "realizar trabalhos de formação a outros colegas de trabalho". Será esse procedimento concertado entre a investigação e a intervenção multifacetada que melhor poderá ajudar "aqueles que ajudam" a retirarem o máximo partido das suas capacidades e a lidarem da melhor forma como a pressão "natural" do trabalho.

\section{Referências}

Blegen, M. A. (1993). Nurses' job satisfaction: A meta-analysis of related variables. Nursing Research, 42, 36-41.

Boice, R., \& Myers, P. E. (1987). Which setting is healthier and happier, academe or private practice? Professional Psychology: Research and Practice, 18, 526-529.

Borda, R. C., \& Norman, I. J. (1997). Factors influencing turnover and absence of nurses: A research review. International Journal of Nursing Studies, 34, 385-394.

Bourbonnais, R., Comeau, M., Vézina, M., \& Guylaine, D. (1998). Job strain, psychological distress, and burnout in nurses. American Journal of Industrial Medicine, 24, 20-28.

Butterworth, T., Carson, J., Jeacock, J., White, E., \& Clements, A. (1999). Stress, coping, burnout, and job satisfaction in British nurses: Findings from the clinical supervision evaluation project. Stress Medicine, 15, 27-33.

Calais, S. L., Andrade, L. M. B., \& Lipp, M. E. N. (2003). Diferenças de sexo e escolaridade na manifestação de stress em adultos jovens. Psicologia: Reflexão e Crítica, 16, 257-263.

Cavalheiro, A. M., Moura Junior, D. F., \& Lopes, A. C. (2008). Estresse de enfermeiros com atuação em unidade de terapia intensiva. Revista Latino-Americana de Enfermagem, 16, 29-35.

Coffey, L. C., Skipper, J. K., \& Jung, F. D. (1988). Nurses and shift work: Effects on job performance and job-related stress. Journal of Advanced Nursing, 13, 245-254.

Cooper, C. L., Dewe, P. J., \& O`Driscoll, M. O. (2001). Organizational stress: A review and critique of theory, research, and applications. London: Sage.

Cooper, C. L., Sloan, S., \& Williams, S. (1988). Occupational Stress Indicator: Management Guide. Windsor: NFER-NELSON.
Cruz, J. F., \& Freitas, M. (1988, Junho). Prevalência e fontes de stress nos professores: um estudo comparativo. Trabalho apresentado na First International Conference on Counselling Psychology and Human Development. Porto, Portugal.

Cruz, J. F., \& Melo, B. M. (1996). Stress e burnou” nos psicólogos: desenvolvimento e características psicométricas de instrumentos de avaliação [manuscrito não publicado]. Universidade do Minho, Braga.

Cruz, J. F., \& Mesquita, A. (1988, Setembro). Incidence and sources of stress in teaching. Trabalho apresentado na $13^{\text {th }}$ Conference of the Association for Teacher Education in Europe. Barcelona, Espanha..

Cunha, R., Cooper, C., Moura, M., Reis, M., \& Fernandes, P. (1992). Portuguese version of the OSI: A study of reliability and validity. Stress Medicine, 8, 247-251.

Douglas, M. K., Meleis, A. I., Eribes, C., \& Kim, S. (1996). The work of auxiliary nurses in México: Stressors, satisfiers, and coping strategies. International Journal of Nursing Studies, 33, 495-505.

Effinger, J., Nelson, L. C., \& Starr, J. M. (1995). Understanding circadian rhythms: A holistic approach to nurses and shift work. Journal of Holistic Nursing, 13, 306-322.

Gil-Monte, P. R. (2002) Influencia del género sobre el proceso de desarrollo del síndrome de quemarse por el trabajo (Burnout) en profesionales de enfermería. Psicologia em Estudo, 7, 3-10.

Gomes, A. R., \& Cruz, J. F. (2004). A experiência de stresse e burnout em psicólogos portugueses: Um estudo sobre as diferenças de género. Psicologia: Teoria, Investigação e Prática, 9, 193-212.

Gomes, A. R., Melo, B., \& Cruz, J. F. (2000). Estudo do stress e do burnout nos psicólogos portugueses. Em J. F. Cruz, A. R. Gomes \& B. Melo (Eds.), Stress e burnout nos psicólogos portugueses (pp. 73-130). Braga: SHO.

Gomes, A. R., Silva, M. J., Mourisco, S., Mota, A., \& Montenegro, N. (2006). Problemas e desafios no exercício da actividade docente: um estudo sobre o stresse, burnout, saúde física e satisfação profissional em professores do $3^{\circ}$ ciclo e ensino secundário. Revista Portuguesa de Educação, 19, 67-93.

Gorsuch, R. L. (1983). Factor analysis (2 ${ }^{\text {nd }}$ ed.). Hillsdale, NJ: Erlbaum.

Hair, J., Anderson, R., Tatham, R., \& Black, W. (1995). Multivariate data analysis with readings $\left(4^{\text {th }} \mathrm{ed}\right.$.). Upper Saddle, NJ: Prentice-Hall.

Hillhouse, J. J., \& Adler, C. M. (1997). Investigating stress effect patterns in hospital staff nurses: Results of a cluster analysis. Social Science \& Medicine, 45, 1781-1788.

Leiter, M. P., \& Harvie, P. (1996). Burnout among mental health workers: A review and research agenda. International Journal of Social Psychiatry, 42, 90-101.

Marshall, N. L., \& Barnett, R. C. (1993). Variations in job strain across nursing and social work specialties. Journal of Community \& Applied Social Psychology, 3, 261-271.

Maslach, C., \& Jackson, S. E. (1996). Maslach Burnout Inventory - Human Services Survey (MBI-HSS). Em C. Maslach, S. E. Jackson \& M. P. Leiter (Eds.), MBI Manual (3 ${ }^{\text {rd }}$ ed.) (pp. 3-17). Mountain View, CA: CPP.

McIntyre, T.M. (1994). Stress e os profissionais de saúde: os que tratam também sofrem. Análise Psicológica, 12, 193-200.

McIntyre, T., McIntyre, S., \& Silvério, J. (1999). Respostas de stress e recursos de coping nos enfermeiros. Análise Psicológica, $17,513-527$ 
Melo, B. T., Gomes, A. R., \& Cruz, J. F. (1997). Stress ocupacional em profissionais da saúde e do ensino. Psicologia: Teoria, Investigação e Prática, 2, 53-71.

Melo, B. T., Gomes, A. R., \& Cruz, J. F. (1999). Desenvolvimento e adaptação de um instrumento de avaliação psicológica do burnout para os profissionais de psicologia. Em A. P. Soares, S. Araújo \& S. Caires (Eds.), Avaliação psicológica: formas e contextos, Vol. VI (pp. 596-603). Braga: APPORT - Universidade do Minho.

Murofuse, N. T., Abranches, S. S., \& Napoleao, A. A. (2005). Reflexões sobre estresse e burnout e a relação com a enfermagem. Revista Latino-Americana de Enfermagem, 13, 255-261.

Narayan, L., Menon, S., \& Spector, P. E. (1999). Stress in the workplace: A comparison of gender and occupations. Journal of Organizational Behavior, 20, 63-73.

Nunnally, J. C. (1978). Psychometric theory (2 ${ }^{\text {nd }}$ ed.). New York: McGraw-Hill.

Parikh, P., Taukari, A., \& Bhattacharya, T. (2004). Occupational stress and coping among nurses. Journal of Health Management, 6, 115-127.

Payne, R., \& Firth-Cozens, J. (1987). Stress in health professionals. New York: Wiley.

Pedhazur, E. (1982). Multiple regression in behavioral research. New York: Holt.

Petterson, I. L., Arnetz, B. B., Arnetz, J. E., \& Horte, L. G. (1995). Work environment, skills utilization, and health of Swedish nurses: Results from a national questionnaire study. Psychotherapy and Psychosomatization, 64, 20-31.

Pinto, A. M. (2000). Burnout profissional em professores portugueses: representações sociais, incidência e preditores. Tese de Doutorado, Universidade de Lisboa, Lisboa, Portugal.
Queirós, P. J. (2005). Burnout no trabalho conjugal em enfermeiros portugueses. Coimbra: Edições Sinais Vitais.

Rodolfa, E. R., \& Kraft, W. A. (1988). Stressors of professionals and trainees at APA-approved counselling and VA medical center internship sites. Professional Psychology: Research and Practice, 19, 43-49.

Sauter, S. L., Hurrel, J. J., \& Cooper, C. L. (1989). Job control and worker health. New York: Wiley.

Shirom, A. (1989). Burnout in work organizations. Em C. L. Cooper \& I. Robertson (Eds.), International review of industrial and organizational psychology (pp. 25-48). New York: Wiley.

Thoresen, R. W., Miller, M., \& Krauskopf, C. J. (1989). The distressed psychologist: Prevalence and treatment considerations. Professional Psychology: Research and Practice, 20, 153-158.

Tummers, G., Merode, F., Landeweerd, A., \& Candel, M. (2003). Individual-level and group-level relationships between organizational characteristics, work characteristics, and psychological work reactions in nursing work: A multilevel study. International Journal of Stress Management, 10, 111-136.

Recebido em 11.04.07

Primeira decisão editorial em 23.07.08

Versão final em 01.12.08

Aceito em 17.12.08 